\title{
$\beta$-Catenin-SOX2 signaling regulates the fate of developing airway epithelium
}

\author{
Shuichi Hashimoto ${ }^{1,2}$, Huaiyong Chen ${ }^{1,2}$, Jianwen Que $^{2}$, Brian L. Brockway ${ }^{1,2}$, Jeffrey A. Drake ${ }^{1,2}$, \\ Joshua C. Snyder ${ }^{1,2}$, Scott H. Randell ${ }^{3}$ and Barry R. Stripp ${ }^{1,2, *}$ \\ ${ }^{1}$ Department of Medicine, Division of Pulmonary, Allergy and Critical Care Medicine, Duke University Medical Center, 106 Research Drive, \\ 2075 MSRBII, DUMC Box 103000, Durham, NC, 27710, USA \\ ${ }^{2}$ Department of Cell Biology, Duke University Medical Center, Box 3709, Durham, NC, 27710, USA \\ ${ }^{3}$ Departments of Cell and Molecular Physiology and Medicine, The University of North Carolina at Chapel Hill, 111 Mason Farm Road, \\ 5200 Medical Biomolecular Research Building, CB 7545 Chapel Hill, NC, 27599-7545, USA \\ *Author for correspondence (barry.stripp@duke.edu)
}

Accepted 11 September 2011

Journal of Cell Science 125, 932-942

(C) 2012. Published by The Company of Biologists Ltd

doi: $10.1242 / j c s .092734$

\section{Summary}

Wnt- $\beta$-catenin signaling regulates cell fate during organ development and postnatal tissue maintenance, but its contribution to specification of distinct lung epithelial lineages is still unclear. To address this question, we used a Cre recombinase (Cre)-LoxP approach to activate canonical Wnt signaling ectopically in developing lung endoderm. We found that persistent activation of canonical Wnt signaling within distal lung endoderm was permissive for normal development of alveolar epithelium, yet led to the loss of developing bronchiolar epithelium and ectasis of distal conducting airways. Activation of canonical Wnt led to ectopic expression of a lymphoid-enhancing factor and a T-cell factor (LEF and TCF, respectively) and absence of SRY (sex-determining region Y)-box 2 (SOX2) and tumor protein p63 (p63) expression in proximal derivatives. Conditional loss of SOX2 in airways phenocopied epithelial differentiation defects observed with ectopic activation of canonical Wnt. Our data suggest that Wnt negatively regulates a SOX2dependent signaling program required for developmental progression of the bronchiolar lineage.

Key words: Wnt, SOX2, Lung development, Endoderm, Bronchiolar epithelium

\section{Introduction}

Complex reciprocal interactions between the endoderm and mesoderm coordinate the process of branching morphogenesis that generates an airway tree in the developing lung (Metzger et al., 2008; Morrisey and Hogan, 2010). During this process, multipotent epithelial progenitor cells proliferate in the distal tips, whereas their daughters undergo lineage restriction to yield specialized cell types of the postnatal lung (Rawlins et al., 2009). The major functionally distinct epithelial cell types that line airways of the mouse lung include basal, secretory and ciliated cells in the pseudostratified bronchial and tracheal airways, nonciliated secretory (Clara) and ciliated cells in the distal conducting (bronchiolar) airways, and type I and II pneumocytes in the alveoli. Early differences in the molecular phenotype of future proximal and distal epithelial lineages include expression of the transcription factors sex-determining region Y (SRY)-box 2 and 9 (SOX2 and SOX9), respectively. However, the signaling pathways that govern these early fate decisions and establishment of specialized epithelial lineages are poorly understood.

The Wnt pathway is a central regulator of organ development and controls diverse cellular functions, such as cell proliferation, differentiation and lineage commitment (Clevers, 2006). Wnt ligands produced by either endodermal and/or mesodermal cells regulate the fate of distal endodermal progenitor cells during the process of lung branching morphogenesis (Goss et al., 2009; Rajagopal et al., 2008; Shu et al., 2002; Wang et al., 2005). Roles for canonical Wnt signaling in this process have been revealed through loss-of-function studies in which the absence of $\beta$ catenin [also known as catenin $\beta 1$ and encoded by Ctnnbl] within early distal lung endoderm results in defects in alveolar development. However, epithelial cells of conducting airways are refractory to the absence of $\beta$-catenin within endodermal progenitors (Mucenski et al., 2003; Zemke et al., 2009). These findings are similar to those in the pancreas in which $\beta$-catenindependent signaling is crucial for development of acinar but not islet epithelium (Murtaugh et al., 2005).

In this study, we used a gain-of-function approach to test the hypothesis that changes in the activity of canonical Wnt signaling have a role in specifying bronchiolar and alveolar lineages. We used a Cre recombinase (Cre)-mediated approach to generate an $\mathrm{N}$-terminally truncated form of $\beta$-catenin that activates canonical Wnt signaling within the developing distal lung endoderm. Our data suggest that high levels of canonical Wnt signaling disrupt the differentiation of bronchiolar lineages but do not affect alveolar development. We identify Sox2 as a key regulator of bronchiolar lineage specification, which is negatively regulated by canonical Wnt signaling.

\section{Results}

Sustained expression of stabilized $\beta$-catenin within early distal lung endoderm disrupts airway patterning

We initially achieved expression of an N-terminally truncated form of $\beta$-catenin ( $\Delta \mathrm{N}-\beta$-catenin) within early distal lung endoderm using a surfactant protein $\mathrm{C}$ (SFTPC)-Cre transgene to 
drive efficient recombination of $\mathrm{Ctnnbl}^{(\mathrm{ex} 3) \text { flox }}$ (supplementary material Fig. S1) and (Okubo et al., 2005). The SFTPC-Cre driver transgene directs recombination of LoxP-flanked (flox) genomic substrates within endodermal lung progenitors, which generate all distal conducting airway and alveolar cell types. Variable low levels of recombination were also observed within epithelial cells of more proximal conducting airway epithelium because descendants of early lung progenitor cells contribute to these structures (supplementary material Fig. S1C,G). SFTPC-Cre(+) mice that were Ctnnbl ${ }^{(e x 3) f l o x / w t}$ heterozygotes (SFTPC-Cre) $\triangle E 3^{\text {het }}$ ) were generated with appropriate Mendelian frequency but showed a high incidence of early postnatal mortality compared with wild-type, SFTPC-Cre or $C$ tnnbl $1^{(e x 3) f l o x}$ mice. Lungs of both embryonic and postnatal SFTPC-Cre/ $\triangle E 3^{\text {het }}$ mice included large dilated airways (Fig. $1 \mathrm{~A}-\mathrm{H}$ ), and pups dying during the early postnatal period showed labored breathing and cyanosis at the time of birth. Mice carrying only SFTPC-Cre were identical to wildtype mice and displayed none of the phenotypic changes observed in SFTPC-Cre/ $\triangle E 3^{\text {het }}$ mice (supplementary material Figs S1, S2). Surviving $S F T P C$-Cre/ $\triangle E 3^{\text {het }}$ pups gained weight in parallel with littermates (wild type, SFTPC-Cre or $C t n n b 1^{(e x 3) f l o x}$ ), although histopathological analysis of mutant animals revealed similar lung lesions to those in embryonic and dying postnatal pups. SFTPC$\mathrm{Cre} / \triangle E 3^{\text {het }}$ animals were fertile, enabling generation of SFTPC$\mathrm{Cre}(+)$ mice that were Ctnnbl $1^{(\text {ex3 } 3) \text { flox/(ex3)flox }}$ homozygotes (SFTPC-Cre/ $\left.\triangle E 3^{\text {homo }}\right)$.

Luminal distension of developing airways in SFTPC-Cre/ $\triangle E 3$ embryos was detected as early as E14.5-E16.5 (compare Fig. 1I and $1 \mathrm{M}$ for E16.5; supplementary material Fig. S1). At later stages, these malformations were confined to the distal regions of the conducting (bronchiolar) airway tree (Fig. 1J-L,N-P). Distal lung parenchyma appeared to be largely normal, with some evidence for local hypoplasia in close proximity to distended bronchiolar airways. Although the number and organization of lung lobes was normal, airway defects were seen in all lobes and in both heterozygous and homozygous SFTPC-Cre/ $\triangle E 3$ mice.

Given that phenotypic changes to lungs of $S F T P C$-Cre/ $\triangle E 3$ mice were restricted to the distal conducting airway without obvious involvement of alveolar or proximal conducting airways, we defined more precisely the spatial pattern of recombination mediated by the SFTPC-Cre transgene. This was initially accomplished by evaluating $S F T P C$-Cre-mediated activation of GFP expression from the (GROSA)26Sor ${ }^{\text {tm } 4 \text { (ACTB-tdTomato-EGFP)Luo } / J}$ (referred to as $R O S A-R G$ ) reporter (supplementary material Fig. $\mathrm{S} 1)$. Expression of GFP, indicative of Cre activity, was seen broadly within most epithelial cells lining the distal tubules of intralobar airways (supplementary material Fig. S1). We then evaluated the spatial expression of $\Delta \mathrm{N}-\beta$-catenin in lungs of conditional mutants to verify that recombination of the $R O S A-R G$ allele was reflective of the spatial domain of $C \operatorname{cnnbl} 1^{(e x 3) f l o x}$ recombination. Lung tissue from SFTPC-Cre/ $\triangle E 3^{\text {homo }}$ mice was evaluated by dual immunofluorescence using primary antibodies recognizing epitopes within either the $\mathrm{N}$ terminus ( $\mathrm{N}$ - $\beta$-cat) or $\mathrm{C}$ terminus (C- $\beta$-cat) of $\beta$ catenin (supplementary material Figs S1, S3). The full-length wildtype form of $\beta$-catenin was detected at the lateral membrane of all airway epithelial cells of $\mathrm{Cre}^{-}$mice (supplementary material Fig. $\mathrm{S} 3 \mathrm{~A}-\mathrm{C}$; and data not shown). By contrast, SFTPC-Cre/AE $3^{\text {homo }}$ lungs contained epithelial cells in distal tubules that stained only for C-terminal $\beta$-catenin at lateral membranes and clusters of epithelial cells with intense cytoplasmic and nuclear anti-C- $\beta$-cat antibody

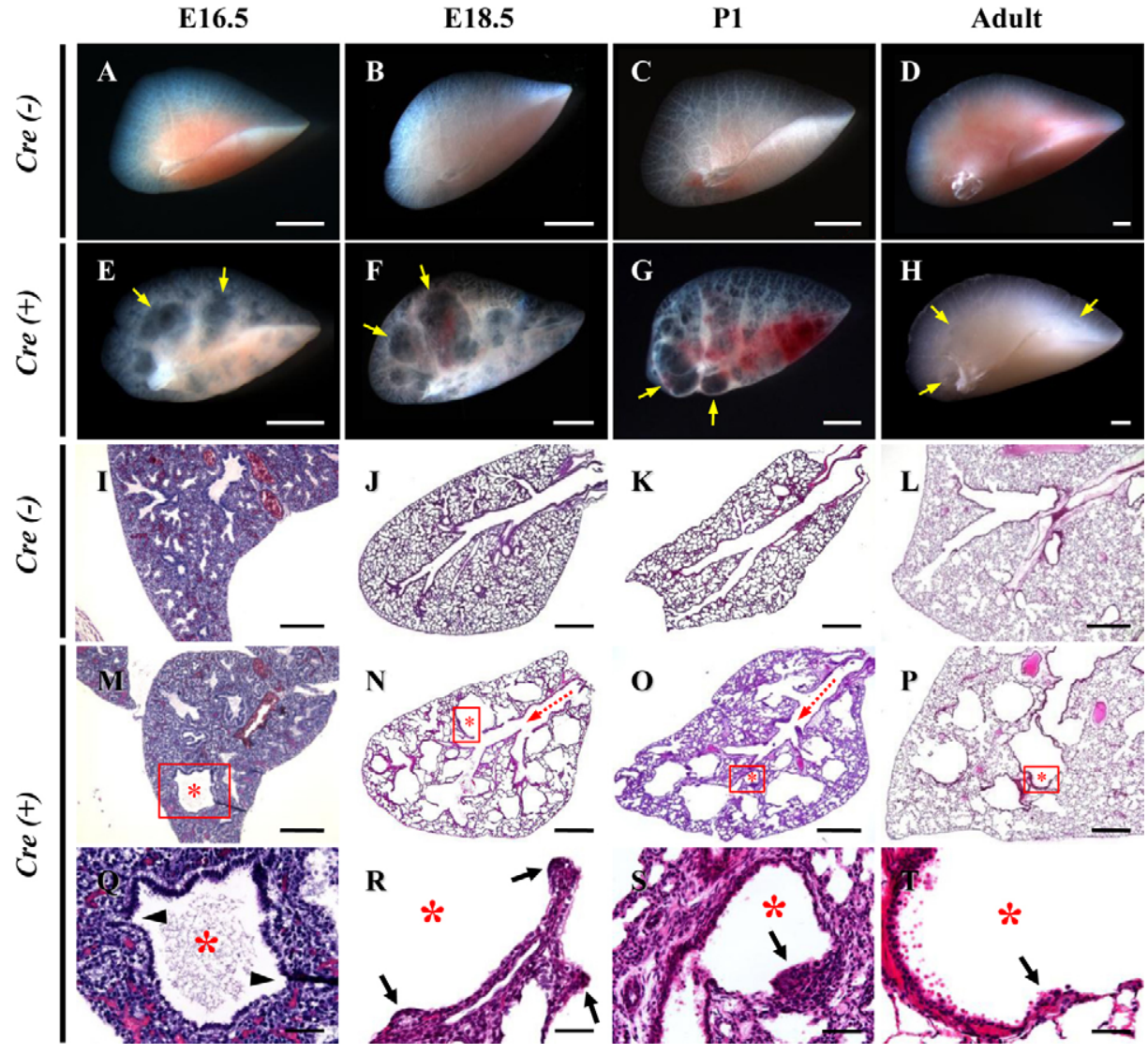

Fig. 1. Developmental abnormalities following ectopic activation of canonical Wnt signaling in developing lung endoderm. Gross appearance and histology of the developing lungs of wildtype Cre(-) (A-D, I-L) and SFTPC-Cre Ctnnbl $1^{(\text {ex } 3) \text { flox }}[\mathrm{Cre}(+)](\mathbf{E}-\mathbf{H}, \mathbf{M}-\mathbf{T})$ mouse lung. Shown are representative images of left lobe and $\mathrm{H}$ and $\mathrm{E}$ stained sections of the indicated genotypes from E16.5 and E18.5, postnatal day 1 (P1) and adult. Arrows in panels E-H identify dilated airways observed in lungs of SFTPC-Cre/ Ctnnbl $1^{(e x 3) f l o x}$ mice. Boxed regions with an asterisk in M-P are shown at higher magnification in Q-T. Arrowheads in Q [ $\mathrm{Cre}(+)]$ at E16.5 show the transition between primitive distal tubular epithelium and distal tip epithelium. Arrows in R$\mathrm{T}$ identify abnormal epithelial hypercellularity in lungs of SFTPC-Cre/Ctnnbl (ex3)flox $^{\text {mice. Scale }}$ bars: $2 \mathrm{~mm}(\mathrm{~A}-\mathrm{H}) ; 200 \mu \mathrm{m}$ (I and M); $500 \mu \mathrm{m}$; (J-L and N-P); $50 \mu \mathrm{m}(\mathrm{Q}-\mathrm{T})$. 
staining but no $\mathrm{N}-\beta$-cat antibody reactivity (supplementary material Fig. S1M-O, arrows; Fig. S3D-I, arrows). These patterns of $\mathrm{C}$ - $\beta$-cat staining without significant $\mathrm{N}$ - $\beta$-cat immunoreactivity confirmed that distal lung epithelium efficiently recombined the $C t n n b 1^{(e x 3) f l o x}$ allele, leading to expression of $\Delta \mathrm{N}-\beta$-catenin. Epithelial clusters showing intense immunoreactivity for $\Delta \mathrm{N}-\beta$-catenin were less numerous within dilated airways of postnatal mice and were only rarely observed within lungs of surviving adult mice (supplementary material Fig. $\mathrm{S} 3 \mathrm{~F}, \mathrm{I})$. By contrast, epithelial cells lining relatively normal alveolar structures of SFTPC-Cre/ $\triangle E 3^{\text {homo }}$ mice showed lower levels of $\Delta \mathrm{N}-\beta$-catenin and expanded postnatally in concert with alveolarization. $\Delta \mathrm{N}-\beta$-catenin expressed within alveolar epithelial cells of adult lungs displayed a nuclear pattern of subcellular localization (supplementary material Fig. S3I, arrowheads).

\section{Stabilized $\beta$-catenin activates transcription of Wnt target genes in developing airways}

We used a $\beta$-galactosidase (Bat-Gal) transgene to assess canonical Wnt signaling in lungs of wild-type and SFTPC-Cre/ $\triangle E 3$ mice. At E14.5, lungs of both Bat-Gal/Cre(-) and Bat-Gall SFTPC-Cre/ $\triangle E 3$ embryos showed intense, patchy, X-Gal staining (Fig. 2A,B). At E16.5 and postnatal day 1 (P1), X-Gal staining was only infrequently observed, with a punctate and apparently random distribution within lungs of Bat-Gal/Cre mice (Fig. 2C,I). By contrast, lungs of E16.5 Bat-Gal/SFTPC$C r e / \triangle E 3$ mice showed robust and broadly distributed X-Gal staining (Fig. 2D) that was reduced but still clearly above wildtype levels at postnatal day 1 (Fig. 2J). This was observed with higher resolution in cryosections from whole-mount X-Galstained lung tissue in which immunohistochemistry for p63 was used to distinguish conducting airway and alveolar epithelium. At E16.5, cells showing X-Gal staining in lungs of Bat-Gal/Cre mice localized to the subepithelial mesenchyme of intrapulmonary bronchial airways (compare Fig. 2E,G with supplementary material Fig. S4A,B). Only a few epithelial cells were positive for X-Gal staining (Fig. 2E, arrowhead). By contrast, robust $\mathrm{X}$-Gal staining was observed among clusters of hyperplastic epithelial cells lining dilated airspaces in distal airways of Bat-Gal/SFTPC-Cre/ $\triangle E 3$ embryos (Fig. 2F,H, arrows). Low levels of X-Gal staining were also observed in focal clusters of epithelial cells lining alveoli of Bat-Gal/SFTPC-Cre/ $\triangle E 3$ lungs (Fig. 2F, arrowhead).

We used p63 immunoreactivity as a marker of conducting airway epithelium and co-stained with X-Gal histochemistry to determine their colocalization. It has been reported that there is p63 immunoreactivity in foregut endoderm before the lungs develop and at high levels within basal cells of adult proximal airways (Que et al., 2007). Here, we used an amplification protocol to demonstrate that cells showing p63 immunoreactivity are present within both bronchial and bronchiolar epithelium of wild-type E16.5 embryos (Fig. 2E,G; supplementary material Fig. S4A,B). No p63 immunoreactivity was observed in epithelial cells lining distal primitive alveolar structures or within nonepithelial cell types (Fig. 2G; supplementary material Fig. S4B; Fig. S5). Furthermore, no p63 immunoreactivity was observed in the simple cuboidal epithelium of $\operatorname{Trp} 63^{-/-}$embryos (Fig. 7A). Dilated bronchiolar airways of Bat-Gal/SFTPC-Cre/ $\triangle E 3$ mice were largely devoid of p63-immunoreactive cells (Fig. 2F,H; supplementary material Fig. S4D,F). Most notable was the absence of p63-immunoreactive cells among recombined

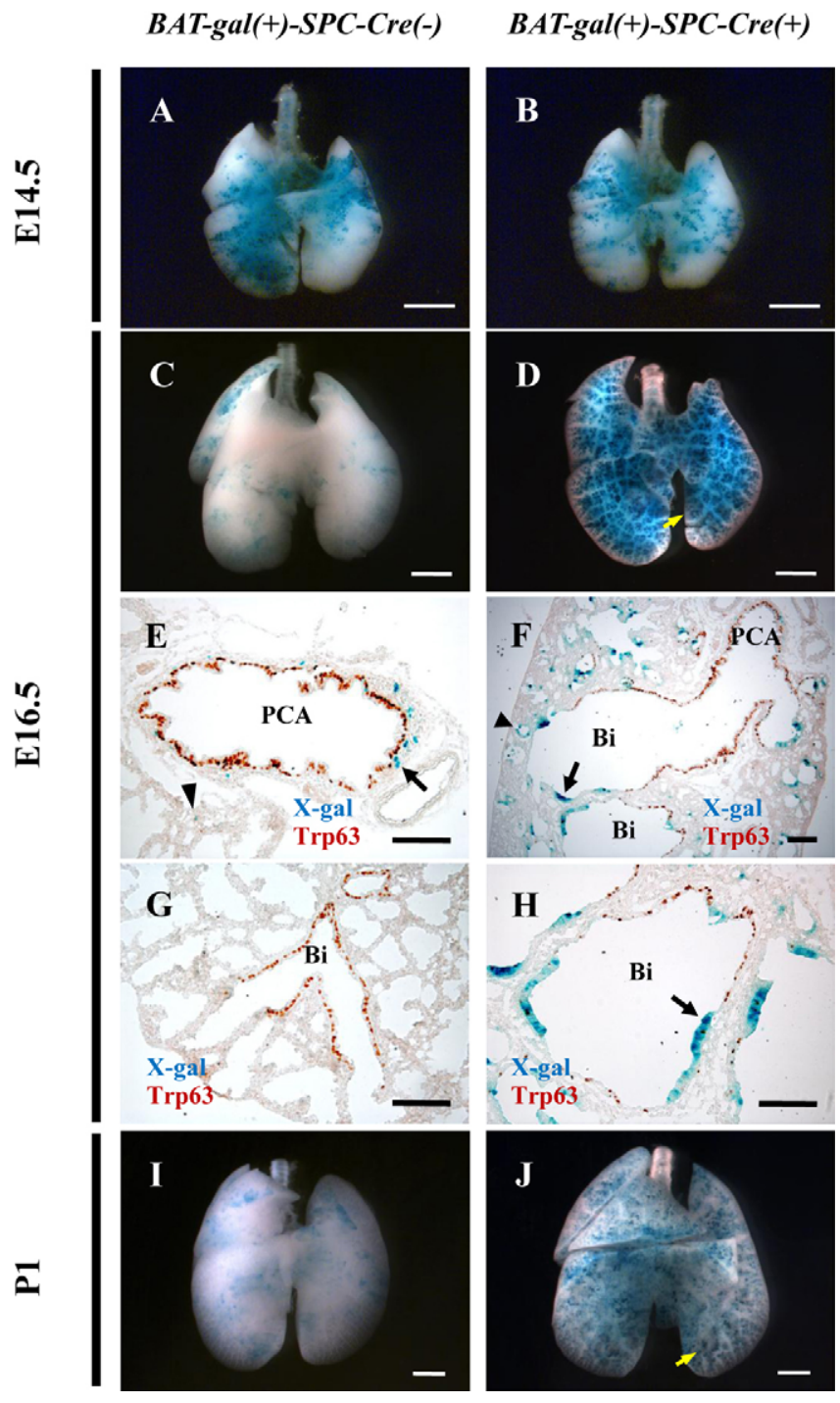

Fig. 2. Ectopic expression of $\Delta \mathbf{N}$ - $\beta$-catenin activates a transgenic reporter of canonical Wnt signaling. Whole-mount X-Gal staining and histology of p63 on X-Gal staining of the developing Bat-gal(+)/SFTPC-Cre/Ctnnb1 $1^{\text {(ex3)flox }}$ mouse lung. Embryos were evaluated at E14.5 and E16.5, and newborn mice were evaluated at postnatal day 1 (P1). Bat-Gal transgene activation is revealed by X-Gal histochemical staining (blue). p63 immunoreactivity is shown as brown DAB staining in E-H. Arrows and arrow heads in E, F and G identify $\mathrm{X}-\mathrm{Gal}$ staining cells in or around conducting airways (arrows) or parenchymal structures (arrowheads). Scale bars: $2 \mathrm{~mm}$ (A-D, I and J); $100 \mu \mathrm{m}(\mathrm{E}-\mathrm{H}) . \mathrm{Bi}$, bronchiole; PCA, proximal conducting airway.

epithelial cells that either were positive for X-Gal staining (Fig. 2F,H, arrow; supplementary material Fig. S4C,D, arrow) or expressed high levels of $\beta$-catenin (supplementary material Fig. S4F, arrowheads).

We evaluated the expression of a LEF and a TCF to confirm that stabilization of $\beta$-catenin led to increased expression of endogenous targets of canonical Wnt signaling. Dual immunofluorescence showed colocalization of C- $\beta$-cat with either Lef1 or Tcf1 within E14.5 lung tissue of wild-type or SFTPC-Cre/ $\triangle E 3$ embryos. In wild-type embryos, LEF1 and TCF1 immunoreactivity was only observed within nuclei of mesenchymal cells surrounding the primitive distal tubular and 
distal-tip epithelium (Fig. 3A-C for LEF1 and 3D-F for TCF1) of developing lungs. This was accompanied by the appearance Bat-Gal transgene activation within some of these mesenchymal cells (Fig. 2E), suggestive of normal activation of the canonical Wnt pathway within these cells. By contrast, robust Lef1 and Tcfl immunoreactivity was observed within recombined epithelium of SFTPC-Cre/AE3 lungs (Fig. 3G-I,J-L, arrows for LEF1 and for TCF1, respectively). Induction of known target genes, together with results of Bat-Gal transgene activation, confirms that truncation of $\beta$-catenin leads to accumulation of the protein and activation of canonical Wnt signaling within some epithelial cells of developing airways.

\section{Differential impact of stabilized $\beta$-catenin on the differentiation of bronchiolar and alveolar epithelium}

We examined the expression of acetylated tubulin (ACT) and secretoglobin, family $1 \mathrm{~A}$, member 1 (Scgbla1) markers for ciliated and secretory cells, and pro-SFTPC and podoplanin (Pdpn; otherwise known as T1 $\alpha$ ), markers of alveolar type 2 and type 1 cells. Wild-type adult lungs contained abundant Scgb1a1positive secretory cells and ACT-positive ciliated cells lining conducting airways (Fig. 4A,B), and Pdpn and pro-SFTPC immunoreactive cells lining alveoli (Fig. 4C,D). SFTPC-Cre/ $\triangle E 3^{\text {homo }}$ lungs contained epithelium of proximal conducting airways that had not undergone recombination and expressed normal levels of $\beta$-catenin, and distal conducting airways that had undergone recombination resulting in expression of high levels of $\Delta \mathrm{N}-\beta$-catenin (Fig. 4E). Nonrecombined epithelial cells of proximal conducting airways in SFTPC-Cre/ $\triangle E 3^{\text {homo }}$ mice contained approximately equal numbers of Scgb1a1-positive secretory cells and ACT-positive ciliated cells (Fig. 4E,F, arrowheads; supplementary material Fig. S6E,F), a characteristic more typical of proximal bronchiolar epithelium of wild-type mice. By contrast, recombined epithelium with strong expression of truncated $\beta$-catenin showed no immunoreactivity for ACT, Scgbla1, pro-SFTPC or Pdpn (Fig. 4E-H, arrows). No expression was seen for other markers of rare cell types, including neuroendocrine cells (calcitonin gene-related peptide) and goblet cells (Muc5ac) in the recombined cells. In contrast to the defects in distal conducting airways, the recombined alveolar epithelium displayed a relatively normal distribution of Pdpn- and pro-SFTPCimmunoreactive alveolar epithelial cells (Fig. 4G,H, red).

Microarray analysis was performed to reveal more comprehensively the nature of changes to the epithelial compartment of SFTPC-Cre/AE3 mice. Fig. 4I displays heat maps that compare transcript levels for clusters of genes representative of bronchial, bronchiolar and alveolar epithelium between total lung RNA isolated from either wild-type or $S F T P C-C r e / \triangle E 3$ mice. Transcripts for genes that are normally expressed preferentially within bronchiolar epithelium (Clara cell markers; cytochrome p450 family members: Cldn10, Fmo1, Pon 1 and Fmo3) were present at levels that were significantly lower in total lung RNA of $S F T P C-C r e / \triangle E 3$ mice relative to wild-type controls. This was accompanied by a significant increase in the relative abundance of gene transcripts for the proximal airway cell markers, Ltf (bronchial gland epithelium), Krt14 and Krt5 (basal cells), and Muc4 (goblet cells). Consistent with elevated expression of basal cell markers, expression of Trp63, a gene expressed at high levels in basal cells of pseudostratified airways and at low levels by epithelial cells of distal conducting airways (Fig. 2), was significantly elevated

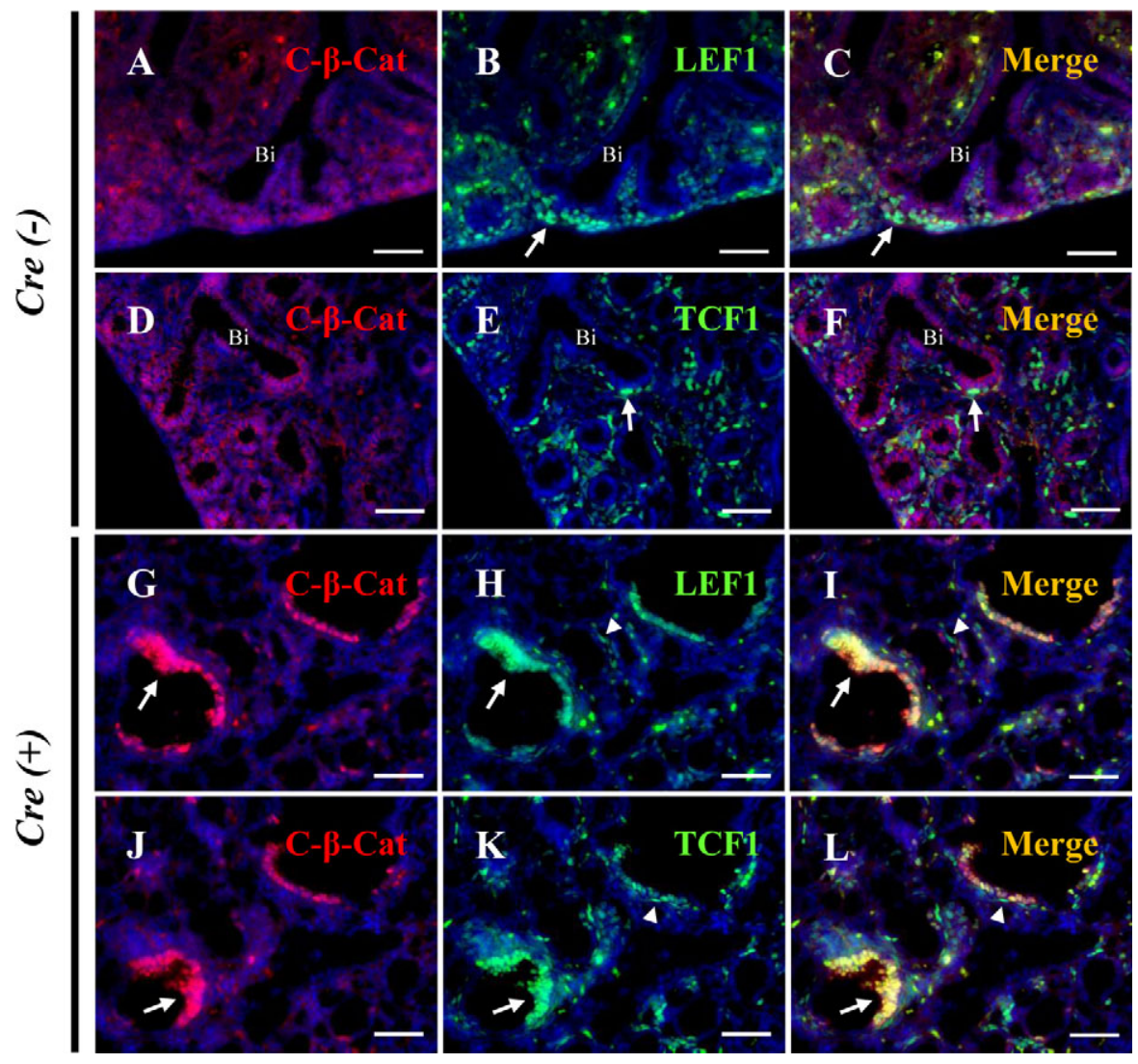

Fig. 3. Ectopic expression of $\Delta \mathrm{N}-\boldsymbol{\beta}$-catenin activates expression of Lef1 and Tef1 factors. $\beta$-catenin, Lef1 and Tcf1 expression in the SFTPCCre/Ctnnbl ${ }^{(e x 3) f l o x}$ mouse lung at E14.5. In the Cre(-) mouse lung at E14.5 (A-F), Lef1 and Tcf1 expression is recognized in mesodermal cells, especially around the distal tubular epithelium (arrow in B,C for Lef1, and arrow in E,F for Tcf1). However, in the $\mathrm{Cre}(+)$ mouse lung at E14.5 (G-L), strong Lef1 and Tcf1 expression is recognized in recombined epithelial cells expressing stabilized $\beta$-catenin [arrow in $\mathrm{G}-\mathrm{I}$ for Lef1 (green) and $\mathrm{C}-\beta$-cat, and arrow in $\mathrm{J}-\mathrm{L}$ for Tcfl and $\mathrm{C}-\beta$-cat]. Arrowheads in $\mathrm{H}-\mathrm{L}$ show unrecombined mesodermal cells expressing Lef1 [arrowhead (green) in H and I] or Tcf1 [arrowhead (green) in $\mathrm{K}$ and L]. Scale bars: $50 \mu \mathrm{m}$. 

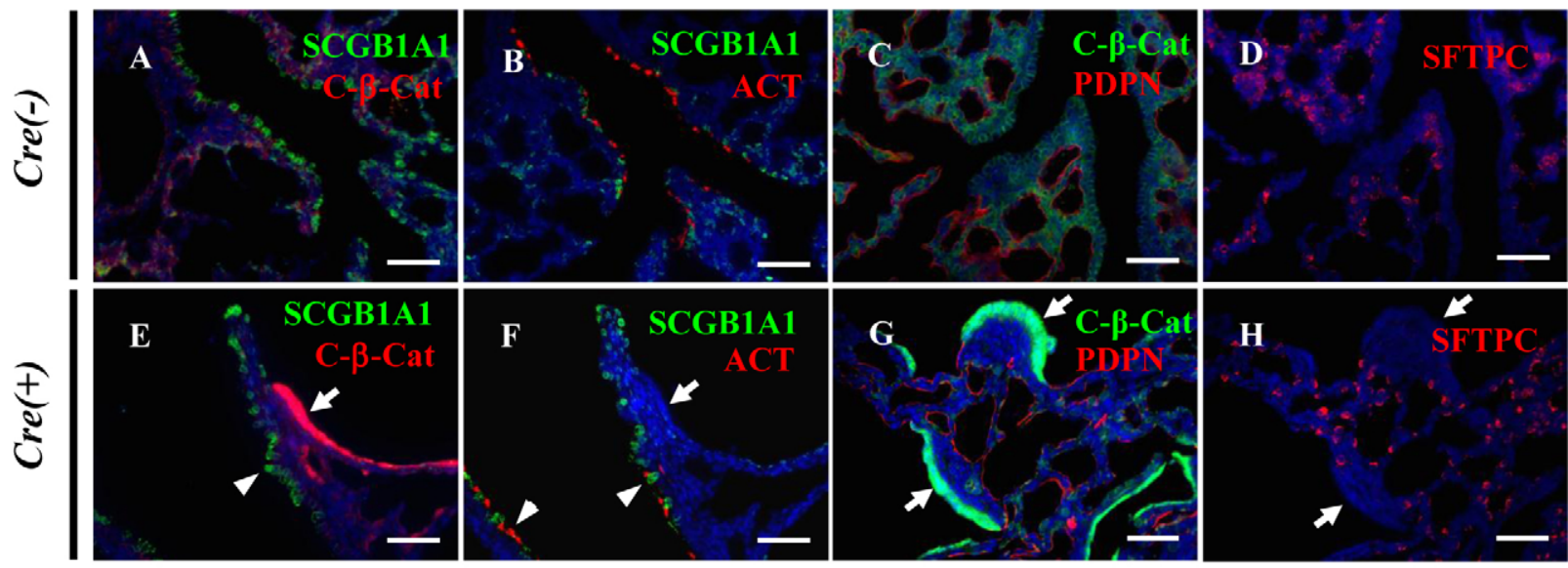

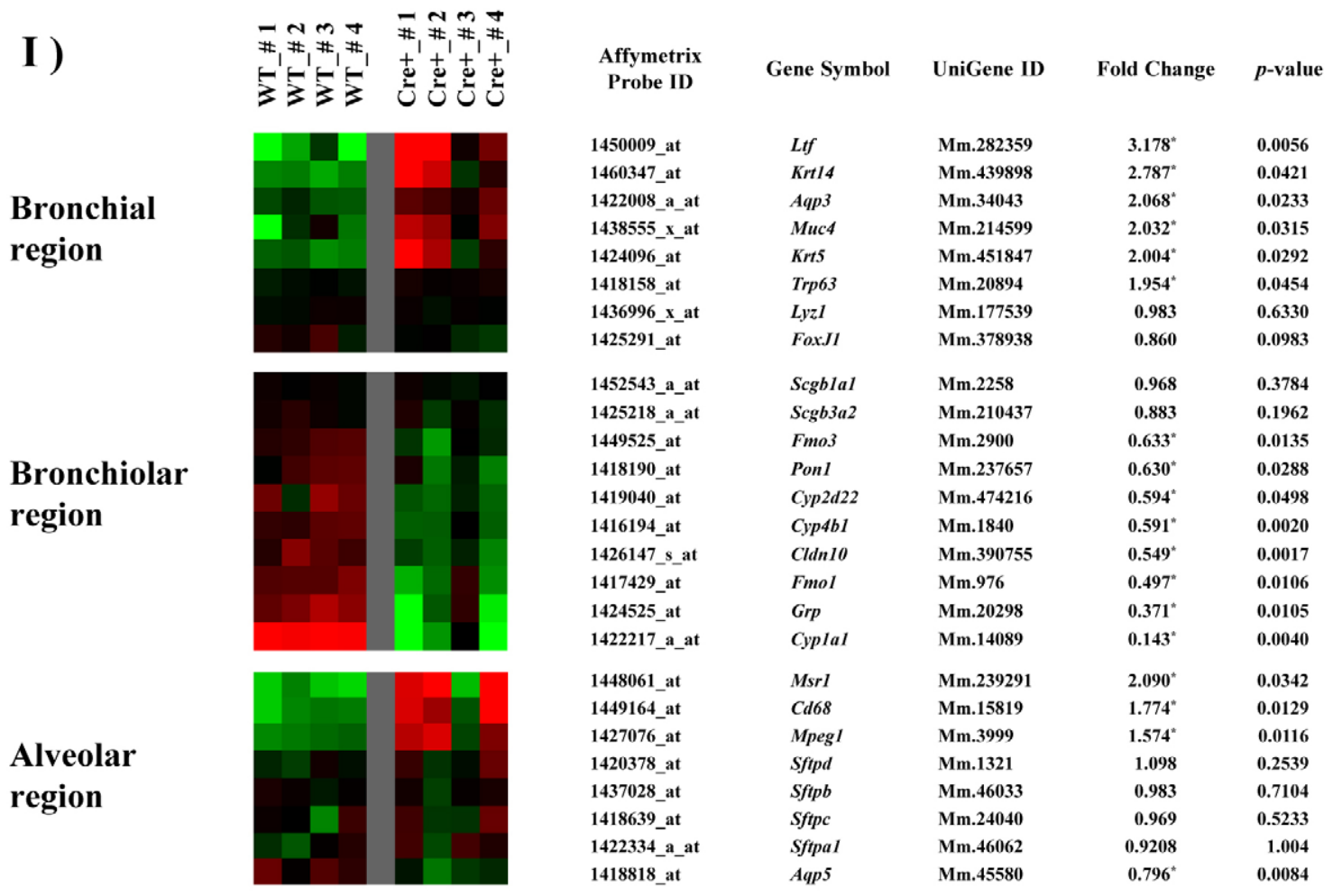

Low expression

High expression

Fig. 4. Prolonged developmental activation of canonical Wnt signaling disrupts bronchiolar epithelial cell differentiation. The expression of epithelial differentiation markers was determined either by immunofluorescence analysis of E18.5 lung tissue or by microarray analysis of total lung RNA from adult mice. Mice were $C \operatorname{tnnb} 1^{(e x 3) f f o x}$ and either positive $[C r e(+)]$ or negative $[C r e(-)]$ for the $S F T P C-C r e$ transgene. Adjacent sections $(\mathbf{A}-\mathbf{B}, \mathbf{C}-\mathbf{D}, \mathbf{E}-\mathbf{F}$ and $\mathbf{G}-\mathbf{H})$ were immunostained for either Scgblal and C- $\beta$-cat (A,F), Scgblal and acetylated tubulin (B,F), C- $\beta$-cat and Pdpn (C,G), or pro-SFTPC (D,H). Arrows in E-H identify recombined epithelial cells expressing $\Delta \mathrm{N}-\beta$-catenin. Arrowheads in E-H identify nonrecombined epithelium. (I) Affymetrix microarray results performed using total RNA from four independent Cre-negative $C t n n b 1^{(e x 3) f l o x}$ (wild-type, WT) mice or four SFTPC-Cre/Ctnnb1 $1^{(e x 3) f l o x}$ mice. Selected genes include those representative of terminal bronchiolar epithelium, including cytochrome p450 families (Cyp 1al, Cyp4b1, Cyp2d22), Fmo1, Cldn10, Pon1, Fmo3, alveolar epithelial cell markers; Sftpa 1, SFTPC, Sftpb, Sftpd and proximal airway cell markers, Ltf (bronchial gland cell marker), Krt14, Krt5, Trp63, Muc4, Lyz1 and Foxj1. Markers of resident or recruited inflammatory cells include Msr1, CD68 and Mpeg1. Fold changes indicated by an asterisk (*) are statistically significant $(P<0.05)$. Scale bars: $50 \mu \mathrm{m}$.

within total lung RNA of SFTPC-Cre/ $\triangle E 3$ mice relative to wildtype controls. By contrast, alveolar type II epithelial cell markers (Sftpal, SFTPC, Sftpb and Sftpd) showed no significant changes.
Genes normally expressed within the alveolar compartment whose transcripts were differentially represented within total lung RNA of wild-type and SFTPC-Cre/AE3 mice included Msrl, 
Cd68 and Mpegl, all of which are expressed by either local or recruited cells of the granulocyte-macrophage lineage. These data are consistent with the observed histopathological analysis suggesting that potentiation of canonical Wnt signaling within early distal lung endoderm selectively disrupts the development of bronchiolar airways.

\section{Impact of stabilized $\beta$-catenin on proliferation and apoptosis within developing bronchiolar and alveolar epithelium}

To test whether recombination affects cell proliferation and/or apoptosis, we assayed apoptotic and proliferating cell activity by immunostaining with antibodies against cleaved caspase-3 and phosphorylated histone 3 (PHH3), respectively (Fig. 5). Immunoreactivity for cleaved caspase-3 was infrequently seen cells of the airway and alveolar epithelium of both wild-type and SFTPC-Cre/ $\triangle E 3^{\text {homo }}$ embryos. However, there were no changes in abundance as a function of $\beta$-catenin activation (Fig. 5A,B).

When cell proliferation was examined, a significant reduction was observed in the number of $\mathrm{PHH} 3$-positive cells within recombined $S F T P C-C r e / \triangle E 3^{\text {homo }}$ epithelium relative to wild type (Fig. 5C-F). No changes were observed in the PHH3-labeling index within epithelium of nonrecombined bronchial airways of SFTPC-Cre/ $\triangle E 3^{\text {homo }}$ mice relative to wild-type controls, suggesting that the reduced proliferative rate of recombined epithelium was a direct consequence of activated $\beta$-catenin signaling (Fig. 5D,F). By contrast, similar PHH3-labeling indices were observed within recombined alveolar epithelium of SFTPC$C r e / \triangle E 3^{\text {homo }}$ mice and alveolar epithelium of wild-type embryos at E18.5 (Fig. 5C-E). These data demonstrate that expression of $\Delta \mathrm{N}-\beta$-catenin directly inhibits the proliferative capacity of developing conducting airway epithelium but has no impact on alveolar epithelial cell proliferation. This result suggests that reduced proliferation specifically of early bronchiolar progenitor cells leads to their dramatic under-representation in the postnatal lung and ectasis of distal conducting airways.

\section{Canonical Wnt signaling downregulates Sox2 and Trp63 expression, leading to defects in the maturation of bronchiolar epithelium}

We asked whether stabilized $\beta$-catenin alters expression of the SRY box transcription factors SOX2 and SOX9; factors that have been used to follow lineage commitment of endodermal progenitor cells (Gontan et al., 2008; Que et al., 2009; Que et al., 2007; Tompkins et al., 2009). Dual immunofluorescence was performed for colocalization of $\mathrm{C}$ - $\beta$-cat with either SOX2 or SOX9 within E14.5 lungs of wild-type or SFTPC-Cre/AE3 embryos. In the wild-type lung, SOX2 was expressed by epithelial cells lining developing airways at all locations with the exception of distal tubular tips (Fig. 6A, arrow). SOX9 was expressed exclusively in the primitive distal tubular tip (Fig. 6B, arrowhead), but was not expressed in more proximal developing airways that express SOX2 (Fig. 6B, arrow). Thus, as shown previously, SOX2 and SOX9 have reciprocal expression patterns in the E14.5 embryonic lung. This pattern was disrupted within recombined airways of E14.5 SFTPC-Cre/ $\triangle E 3$ embryos. SOX2 expression was absent (Fig. 6D,E, arrows) and SOX9 expression was present within recombined epithelium (Fig. 6G,H, arrows). By contrast, nonrecombined epithelial cells within conducting airways and the recombined epithelium of alveolar airways displayed normal expression of SOX2 and SOX9, respectively.
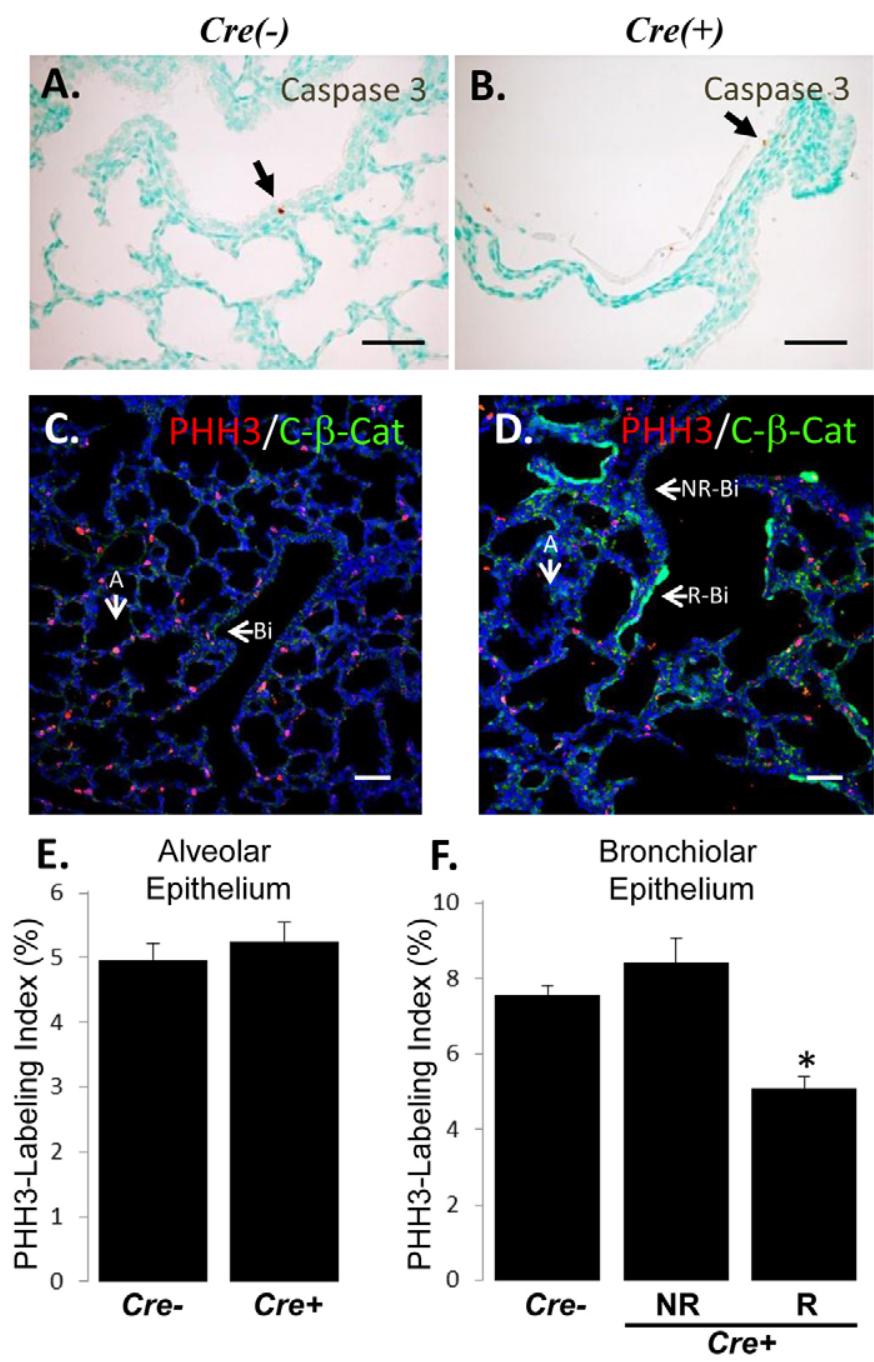

Fig. 5. Ectopic activation of canonical Wnt decreases epithelial cell proliferation but has no impact on apoptosis. Immunostaining for caspase-3 and $\mathrm{PHH} 3$ was performed on E18.5 lung tissue from $C t n n b 1^{(e x 3) f l o x}$ mice that were either positive $[\mathrm{Cre}(+)]$ or negative $[\mathrm{Cre}(-)]$ for the SFTPC-Cre transgene. $(\mathbf{A}, \mathbf{B})$ Photomicrographs illustrating positive DAB immunostaining (brown) with methyl green counterstain to reveal nuclei. Arrows identify rare caspase-3positive cells. (C,D) Photomicrographs revealing PHH3 (red) and C- $\beta$-cat (green) immunoreactivity. (E,F) Bar graphs showing results of morphometry ( $n=3$ E18.5 embryos per group) to determine PPH3-labeling index of the indicated epithelial compartment of wild-type [Cre(-)] and SFTPC-Cre/ $C$ tnnbl $1^{(e x 3) f l o x}$ mouse lung tissue. Scale bars: $50 \mu \mathrm{m}$. A, alveolus; Bi, bronchiole; NR-Bi, nonrecombined bronchiolar epithelium of $\mathrm{Cre}(+)$ lungs; R$\mathrm{Bi}, \mathrm{Catnn}^{(\text {ex3) }}$-activated bronchiolar epithelium of $\mathrm{Cre}(+)$ lungs. $* P<0.01$.

These data are consistent with previous reports suggesting that SOX9 is a downstream target of canonical Wnt signaling (Blache et al., 2004) and that altered expression of SOX factors contributes to defects in development of bronchiolar airways following ectopic activation of canonical Wnt signaling.

Given that neither SOX2 (Fig. 6) nor p63 (Fig. 2; supplementary material Fig. S4) were expressed within developing recombined epithelium of SFTPC-Cre/ $\triangle E 3$ embryos, we examined the interdependency of their expression and their functional roles in regulating epithelial maturation in developing airways. Previous 

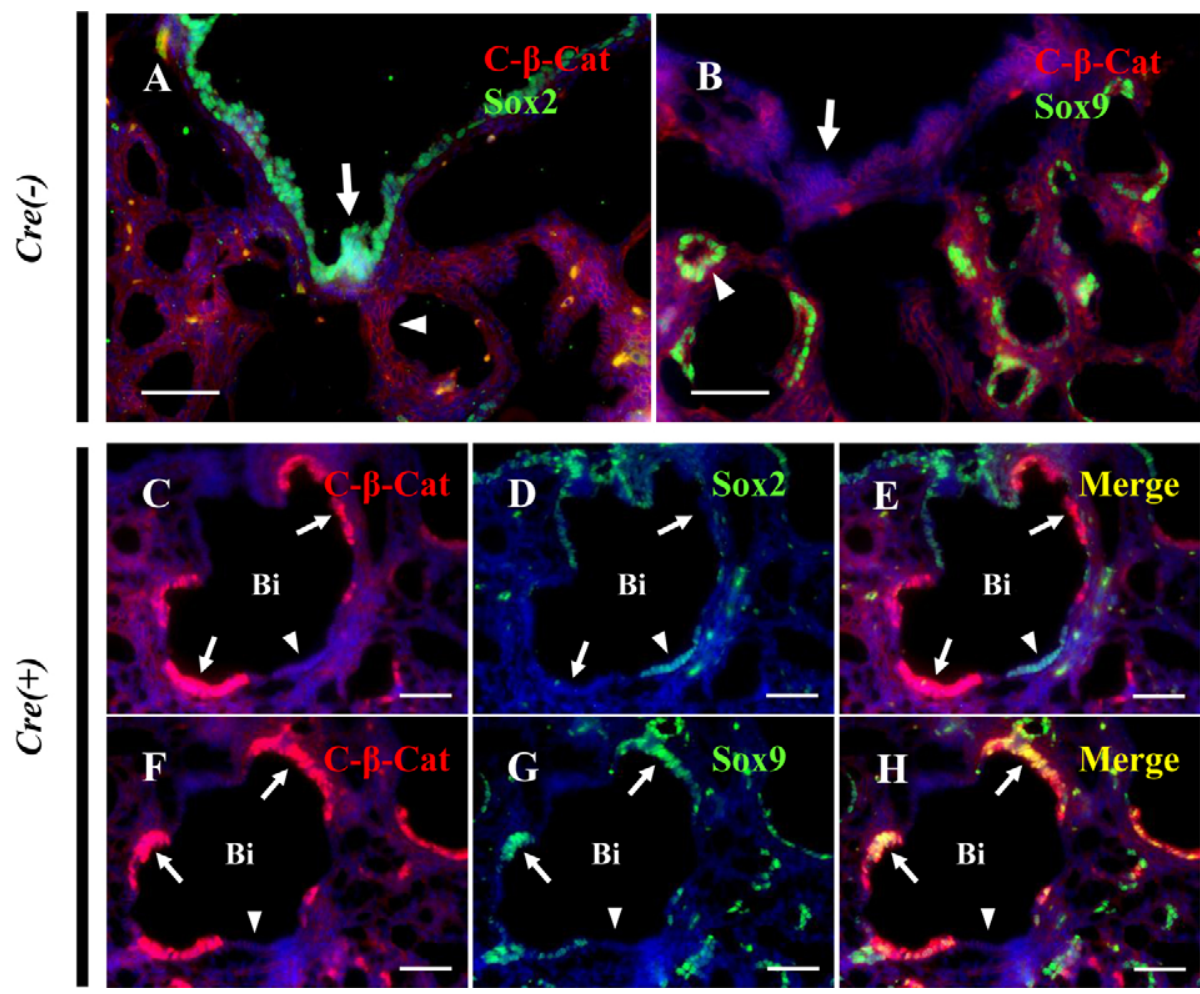

Fig. 6. Activation of canonical Wnt signaling alters spatial expression of SOX2 and SOX9 in developing airways. Immunofluorescence detection of C- $\beta$-cat, SOX2 and SOX9 in E14.5 lung tissue from $C t n n b 1^{(\text {ex } 3) \text { flox }}$ mice that were either positive $[\mathrm{Cre}(+)]$ or negative $[\mathrm{Cre}(-)]$ for the SFTPC-Cre transgene. For all panels, $\beta$-catenin immunoreactivity is in red fluorescence and either SOX2 $(\mathbf{A}, \mathbf{D}, \mathbf{E})$ or SOX9 $(\mathbf{B}, \mathbf{G}, \mathbf{H})$ is indicated by green fluorescence. Arrows and arrowheads in A and B identify proximal and distal lung structures, respectively. Arrows and arrowheads in

$\mathbf{C}-\mathbf{H}$ identify recombined and nonrecombined proximal airway epithelium, respectively. Scale bars: $50 \mu \mathrm{m}$. Bi, bronchiole.

studies demonstrated that either p63 deficiency or conditional loss of SOX2 in the developing lung results in aberrant epithelial cell maturation within tracheobronchial epithelium (Daniely et al., 2004; Que et al., 2009; Tompkins et al., 2009). We initially asked whether p63 deficiency has an impact on the expression of SOX2 in E17.5-18.5 lung tissue of $\operatorname{Trp}^{-/-}$embryos. Double immunofluorescence staining revealed that airways deficient in p63 express normal levels of SOX2 (Fig. 7A-C). By contrast, analysis of $N k x 2.5-C r e(+) / S o x 2^{\triangle C O N D / \triangle C O N D}$ embryonic lungs revealed that SOX2-deficient airways lacked p63 (Fig. 7D-F). These data suggest that SOX2 is an upstream regulator of p63 in developing airway progenitor cells. Moreover, the inability to upregulate SOX2 expression in distal lung endoderm helps to explain defects in the development of bronchiolar airway lineages in the setting of ectopic Wnt activation.

\section{Discussion}

\section{Ectopic Wnt signaling disrupts bronchiolar development}

The Wnt pathway controls many aspects of lung development (Goss et al., 2009; Li et al., 2002; Zhang et al., 2008). However, its role in epithelial differentiation during the stage of branching morphogenesis is poorly understood (Goss et al., 2009; Li et al., 2002; Mucenski et al., 2005; Shu et al., 2005). Our data suggest that prolonged activation of canonical Wnt signaling within distal endoderm progenitors in the early developing lung has a differential impact on their differentiation into bronchiolar versus alveolar lineages. Specifically, expression of constitutively active $\Delta \mathrm{N}$ $\beta$-catenin resulted in defective bronchiolar epithelial cell differentiation and marked ectasis of the developing and adult airway. By contrast, the generation and differentiation of alveolar structures appears to be refractory to the effects of elevated $\Delta \mathrm{N}$ $\beta$-catenin. These findings suggest that ectopic activation of canonical Wnt signaling within developing distal lung progenitors yields proximal derivatives that fail to assume normal conducting airway epithelial cell fates.

\section{Canonical Wnt signaling is incompatible with SOX2 expression in developing airway epithelium}

Epithelial cells of the early lung endoderm are multipotent, having the capacity to generate either conducting airway or alveolar lineages of the postnatal lung (Rawlins et al., 2009; Shannon, 1994). Early distinctions between endodermal progenitors that give rise to alveolar or conducting airway structures of the late embryonic and postnatal lung include the relative activity of Wnt signaling and expression of either SOX9 (distal tip endoderm and/or alveolar) or SOX2 (proximal endoderm and/or conducting airway). Loss-of-function studies have shown that $\beta$-catenin has a crucial role in the specification of progenitor cells that give rise to alveolar epithelium of the postnatal lung (Mucenski et al., 2003). Here, we examined the effect of continued high levels of canonical Wnt signaling, achieved through activation of $\Delta \mathrm{N}$ - $\beta$-catenin in developing lung endoderm. This resulted in ectopic expression of Lef1 and Tcf1, and activation of a Wnt responsive transgenic reporter (Bat-Gal), thus confirming activation of canonical Wnt signaling. Ectopic activation of canonical Wnt signaling expanded the domain of SOX9 expression to include recombined cells within dilated airways. These SOX9-expressing epithelial cells were SOX2negative but failed to assume characteristics of differentiating lung epithelium. These findings are consistent with other work demonstrating expression of SOX9 and canonical Wnt activation within the undifferentiated epithelium residing within the distaltip domain of early developing lung endoderm (Okubo and Hogan, 2004). Similar expansion of the domain of SOX9 expression and defects in epithelial differentiation observed in conditional SOX2-null airways further support a link between 


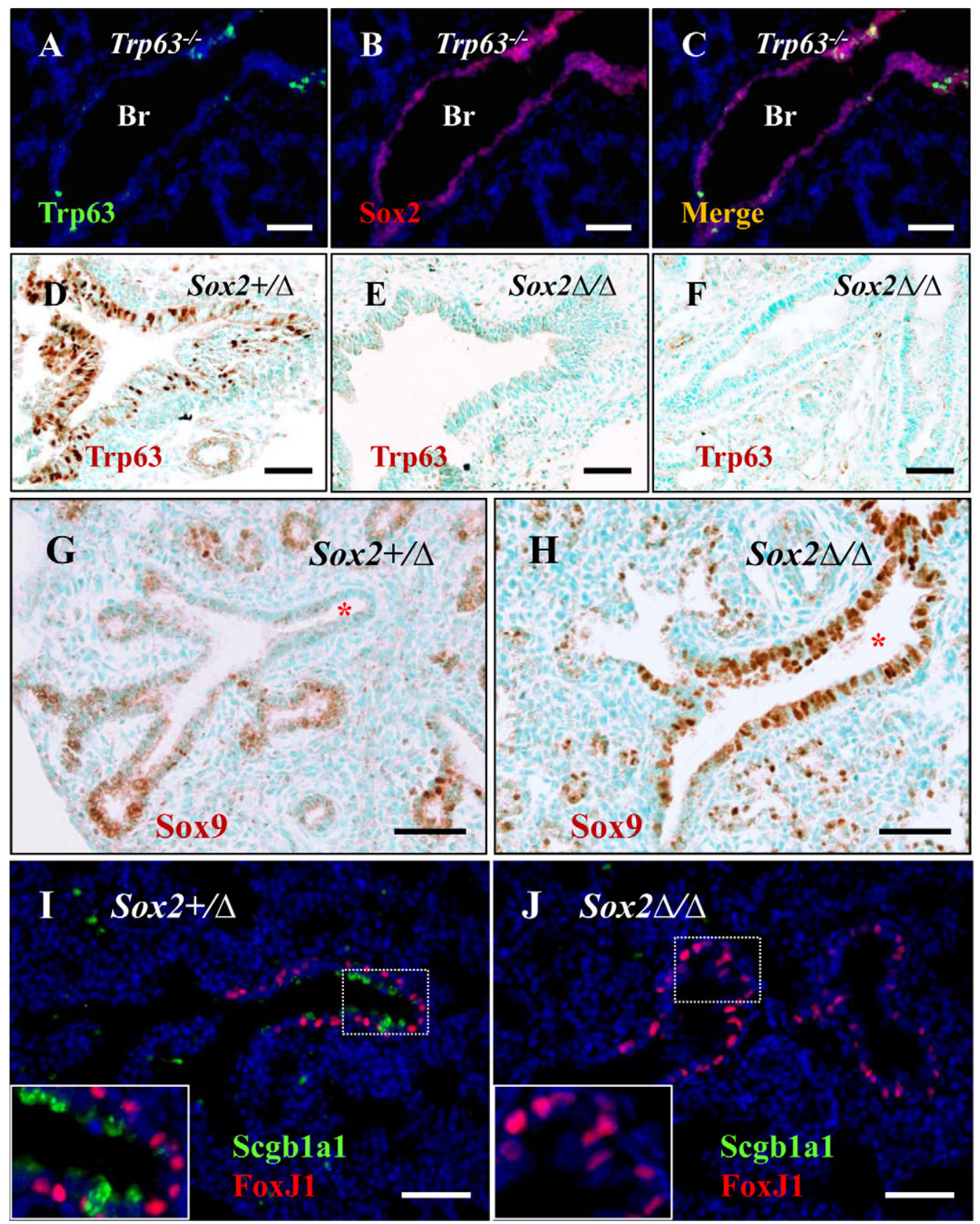

Fig. 7. Conditional loss of SOX2 in airway epithelium phenocopies epithelial differentiation defects seen with ectopic Wnt. (A-C) SOX2 expression in the E17.5 lung of $\operatorname{Trp} 63^{\mathrm{Brdm} 2 / \mathrm{Brdm} 2}\left(\operatorname{Trp} 63^{-/-}\right)$mice. p63 and SOX2 expression is visible as green and red immunofluorescence, respectively.

(D-F) Immunohistochemical detection of p63 in E14.5 proximal airways of $\operatorname{Sox}^{+/ \Delta}$ or $\operatorname{Sox} 2^{\Delta / \Delta}$ lung. (G-H) Immunohistochemical localization of SOX9 in distal epithelium of lung from $\operatorname{Sox}_{2} / \Delta$ or Sox $2^{\Delta / \Delta}$ E18.5 embryos. In D-H, positive staining is shown in brown with methyl green counterstaining. (I,J) Immunofluorescent localization of FoxJ1 (ciliated cells; red fluorescence) and Scgb1a1 (secretory cells; green fluorescence) in lung tissue from $\operatorname{Sox}^{+/ \Delta}$ or $\operatorname{Sox}^{\Delta / \Delta}$ E18.5 embryos. Scale bars: $50 \mu \mathrm{m}$.

Wnt-dependent inhibition of SOX2 expression and bronchiolar defects in lungs of SFTPC-Cre/ $\triangle E 3$ mice. These data suggest that appropriate temporal and spatial downregulation of canonical Wnt is necessary for SOX2 expression, commitment to a proximal lineage and generation of specialized epithelial cells of bronchiolar airways. Our finding that p63 is a downstream target of SOX2 expression suggests that loss of p63 following ectopic activation of canonical Wnt signaling contributes to epithelial cell differentiation defects observed in SFTPC-Cre/ $\triangle E 3$ mice.

Development of a normal alveolar epithelium from distal tip cells in SFTPC-Cre/ $\triangle E 3$ mice could indicate either that regional progenitors are refractory to ectopic $\beta$-catenin stabilization, or that a subpopulation of nonrecombined cells selectively undergoes alveolar lineage differentiation. The finding that most of the developing distal epithelium and postnatal alveolar epithelium was recombined and expressed truncated $\beta$-catenin, yet expressed typical markers of specialized alveolar epithelial cells, suggests that alveolar lineage differentiation can proceed despite ectopic canonical Wnt activation.

\section{Distinct developmental stage- and cell-type-specific effects of Wnt signaling}

The defects observed in the establishment and differentiation of bronchiolar airways following ectopic expression of $\Delta \mathrm{N}-\beta$ catenin in early lung endoderm contrast with our previous findings using a similar strategy to express $\Delta \mathrm{N}$ - $\beta$-catenin within bronchiolar epithelial cells of late embryonic airways (Reynolds et al., 2008). In this earlier study, ectopic activation of canonical Wnt within conducting airway epithelium of late gestation 
embryos arrested maturation of bronchiolar progenitor cells but did not alter their long-term maintenance or proliferative capacity in the postnatal period. Taken together, these results reveal developmental stage-specific consequences of expressing $\Delta \mathrm{N}-\beta$ catenin in lung endoderm. Our data demonstrating Bat-Gal transgene activation following expression of $\Delta \mathrm{N}-\beta$-catenin within early lung endoderm suggest that members of the LEF-TCF family of $\beta$-catenin-interacting proteins are co-expressed at this developmental stage, leading to potentiation of canonical Wnt signaling. This occurs despite the relative inactivity of Wnt reporter transgenes within the proximal endoderm of developing wild-type lung (Okubo and Hogan, 2004; Reynolds et al., 2008; Zhang et al., 2008).

The importance of canonical Wnt signaling for development of the alveolar epithelium has been shown in mouse models in which conditional $\beta$-catenin deletion results in loss of alveolar structures (Mucenski et al., 2003). Despite this crucial role for $\beta$ catenin in alveolar development, its deletion within epithelial progenitor cells of developing conducting airways has no impact on airway patterning, epithelial differentiation or in the maintenance of epithelial progenitor cells that maintain adult airways (Mucenski et al., 2003; Zemke et al., 2009). These reports contrast with our current data showing that ectopic activation of $\beta$-catenin within developing lung endoderm is permissive for alveolar development yet profoundly disrupts bronchiolar development. Our data suggest that committed bronchiolar progenitor cells express members of the LEF-TCF family of $\beta$-catenin-interacting proteins and that their heterodimerization with ectopically stabilized $\beta$-catenin leads to the activation of canonical Wnt signaling. By contrast, alveolar progenitor cells fail to activate the canonical Wnt pathway despite expression of an activated form of $\beta$-catenin, thus explaining the permissive nature of stabilized $\beta$-catenin for developmental progression of this lineage. Expression of both Lefl and Tcf4 have been demonstrated within developing airways of the mouse lung and have important roles in the formation of submucosal gland structures in proximal airways (Driskell et al., 2007; Duan et al., 1999). Interestingly, TCF factors have been shown in the epidermis to exert effects on gene transcription and cellular differentiation in a $\beta$-catenin-independent fashion (Nguyen et al., 2009). Although expression of LEF and TCF are thought to represent mediators of canonical Wnt signaling in the development of submucosal glands (Driskell et al., 2007), our data suggest that $\beta$-catenin-independent functions of LEF and TCF regulate the behavior of early bronchiolar progenitors and that these functions are disrupted by ectopic stabilization of $\beta$ catenin.

Other Cre driver lines have been used for activation of the Ctnnb1 ${ }^{(e x 3) f l o x}$ allele in the developing foregut and early lung endoderm and have led to defects in the patterning of branching airways and differentiation of proximal epithelial lineages (Goss et al., 2009; Li et al., 2009; Zhang et al., 2008). We did not observe patterning or differentiation defects in proximal conducting airways of SFTPC-Cre/AE3 mice. We attribute differences in outcome between our study and others to the efficiency of recombination within distinct airway compartments. Although efficient recombination of the Ctnnbl ${ }^{(e x 3) f l o x}$ allele occurred in presumptive bronchioles and distal tip endoderm of SFTPC-Cre/ $\triangle E 3$ mice, inefficient recombination in endodermal progenitors of the postnatal tracheobronchial epithelium precluded analysis of $\Delta \mathrm{N}-\beta$-catenin expression in this airway compartment.

The phenotype of SFTPC-Cre/AE3 mice differs from that obtained using the same SFTPC promoter to drive ectopic expression of a Lef1- $\beta$-catenin fusion protein (Okubo and Hogan, 2004). Expression of Lef1- $\beta$-catenin within distal developing lung endoderm leads to downregulation of lung epithelial markers and activation of genes normally associated with secretory cells of the intestinal epithelium. An important difference between these studies is that the present findings rely on endogenous co-expression of LEF-TCF factors for activation of canonical Wnt signaling following expression of $\Delta \mathrm{N}-\beta-$ catenin. Although developing bronchioles in SFTPC-Cre/ $\triangle E 3$ mice displayed robust Bat-Gal transgene activation, we found varying levels of Bat-Gal activation in distal tip epithelium despite relatively uniform persistent expression of $\Delta \mathrm{N}-\beta$-catenin. We speculate that differential responses to $\Delta \mathrm{N}-\beta$-catenin versus a Lef1- $\beta$-catenin fusion protein reflect variable levels of endogenous TCF-LEF factor expression.

\section{Mechanism of airway ectasis and implications for lung disease}

The observations reported here add to the collective body of information about the role of Wnt signaling at different stages, and within distinct cell types, of the developing lung. They suggest that tightly regulated Wnt signaling during later stages of lung development are crucial for appropriate specification of distinct epithelial lineages. We also define SOX2 as a crucial regulator of fate decisions in the early developing lung and whose expression is disrupted by ectopic activation of canonical Wnt. The selective loss of bronchiolar lineages and the continued proliferation of alveolar progenitors resulted in airway ectasis in postnatal survivors. In humans, similar developmental anomalies are collectively referred to as 'congenital cystic adenomatous malformations' (CCAM) (Morotti et al., 1999). We speculate that these developmental anomalies result from signaling defects at an early stage in lung development when distinct epithelial lineages are specified. Further studies are needed to reveal signaling defects and target cell types whose dysregulation culminates in human CCAM. However, our studies point to Wnt and SOX2 signaling and early events in the specification of alveolar versus airway lineages as possible targets.

\section{Materials and Methods}

\section{Animal husbandry}

Mice were maintained in an Association for Assessment and Accreditation of Laboratory Animal Care (AAALAC)-certified institutional vivarium. All experimental procedures were approved by the Institutional Animal Care and Use Committee (IACUC) of either the University of Pittsburgh or Duke University.

\section{Transgenic mice and embryos}

Mice carrying a mutant allele of the $\beta$-catenin $(C t n n b 1)$ gene in which loxP sites flank exon 3 (Ctnnbl $\left.{ }^{(e x 3) f l o x}\right)$, and transgenic mouse lines expressing Cre under control of the human SFTPC promoter, have been described previously (Harada et al., 1999; Okubo et al., 2005; Reynolds et al., 2008). The Ctnnbl ${ }^{(\text {ex } 3) f l o x}$ mice were bred with the SFTPC-Cre driver line for the generation of lines that were either heterozygous or homozygous for $\mathrm{Ctnnbl}^{(e x 3) f l o x}$. Mendelian transmission of SFTPC-Cre and Ctnnbl ${ }^{(e x 3) f f o x}$ was only observed when the two loci were transmitted through the female germline. Paternal transmission of SFTPC-Cre resulted in germline recombination of co-transmitted floxed alleles (data not shown) and embryonic lethality of mice receiving the recombined paternal allele of Ctnnbl $1^{(e x 3) f l o x}$. This line was bred to mice harboring the Bat-Gal transgene [B6.Cg-Tg(Bat-lacZ)3Picc/J, Jackson Laboratories, Bar Harbor, ME] to create animals that were heterozygous for Ctnnbl ${ }^{(e x 3) f l o x}$ and hemizygous for both 
SFTPC-Cre and Bat-Gal transgenes. Mice carrying a conditional null allele of the Sox2 gene $\left(\operatorname{Sox} 2^{C O N D}\right)$ were a gift from Larysa Pevny, UNC-Chapel Hill (Taranova et al., 2006). The Nkx2.5-Cre mouse line was generated by Robert Schwartz

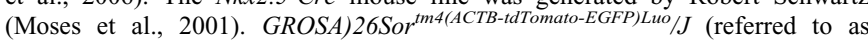
$R O S A-R G)$ was obtained from Jackson Laboratories. $N k x 2.5$-Cre/Sox $2^{+/ 4}$ $\left(\operatorname{Sox}_{2}{ }^{+/ 4}\right)$ and $\mathrm{Nkx2.5-Cre/Sox2^{4/4 }}\left(\operatorname{Sox}^{4 / 4}\right)$ mice were generated as described previously (Que et al., 2009). Mice heterozygous for the Trp $63^{B r d m 2}$ null allele (Mills et al., 1999) were purchased from Jackson Laboratories. Homozygous $\operatorname{Trp} 63^{B r d m 2 / B r d m 2}$ (hereon referred to as $\operatorname{Tr} p 63^{-/}$) mice were generated by crossing heterozygotes and identifying homozygotes based upon external phenotype (absence of limbs and translucent epidermis). Genotyping for each line was by PCR amplification of genomic DNA.

\section{Tissue processing}

Lungs were processed for RNA isolation and/or histological analysis using standard methods as described previously (Reynolds et al., 2008). Briefly, lung tissue was fixed through instillation of $10 \%$ neutral buffered formalin (NBF) followed by immersion in $10 \% \mathrm{NBF}$ for a total of 2 hours at $4^{\circ} \mathrm{C}$. For histochemical detection of $\beta$-galactosidase enzymatic activity, tissues were incubated in X-Gal staining solution $\left[5 \mathrm{mmol} / \mathrm{L} \quad \mathrm{K}_{3} \mathrm{Fe}_{3}, 5 \mathrm{mmol} / \mathrm{L} \quad \mathrm{K}_{4} \mathrm{Fe}_{6}\right.$, $2 \mathrm{mmol} / \mathrm{L} \mathrm{MgCl}_{2}, 0.02 \%$ Nonidet $\mathrm{P}-40,0.01 \%$ sodium deoxycholate, $1 \times \mathrm{PBS}$, and $1 \mathrm{mg} / \mathrm{ml} \mathrm{X-Gal,} \mathrm{for} 4-5$ hours at $37^{\circ} \mathrm{C}$ in the dark, washed in PBS and postfixed in $10 \% \mathrm{NBF}$ at $4{ }^{\circ} \mathrm{C}$ overnight

\section{Immunofluorescence and immunohistochemistry}

Three-color immunofluorescence staining was conducted using the following primary and secondary antibodies. Primary antibodies: goat or rabbit anti-Scgbla1; 1:10,000, (Stripp et al., 2002), mouse $\mathrm{IgG}_{2 \mathrm{~b}}$ anti-acetylated Tubulin (ACT) (1:12,000, Sigma-Aldrich), Rabbit anti-pro-Surfactant Protein C (SFTPC) (1:2500, a gift from Peter Di, University of Pittsburgh), hamster anti-Pdpn [1:30, \# 8.1.1, Developmental Studies Hybridoma Bank (DSHB) at the University of Iowa] rabbit anti-SOX2 (1:1500 Seven Hills Bioreagents, Cincinnati, OH), rabbit antiSOX9 (1:1000 Millipore), mouse $\operatorname{IgG}_{2 \mathrm{a}}$ anti-p63 (4A4; $1: 250$, Santa Cruz Biotechnology, Inc, Santa Cruz, CA), rabbit anti-N-terminus $\beta$-catenin (1:400, Cell Signaling Technologies, Inc., Danvers, MA), mouse $\operatorname{IgG}_{1}$-anti-C-terminus $\beta$ catenin (1:400, BD Transduction Laboratories, San Jose, CA), rabbit anti-Lef1 (1:100, Cell Signaling Technologies), rabbit anti-Tcf1 (1:100, Cell Signaling Technologies), rabbit anti-calcitonin gene-related peptide (Claca; formerly known as CGRP) (1:5000, Sigma-Aldrich), mouse $\operatorname{IgG}_{1}$ anti-Muc5ac (1:200, Lab Vision Inc, Fremont, CA), rabbit anti-Trefoil Factor 3 (Tff3) (1:2,000, a gift from Werner Hoffmann, Otto-von-Guericke-University, Germany), Rabbit anti-cleaved Caspase-3 (Asp175) and rabbit anti-phosphohistone H3 (Ser 10) (1:300, Cell Signaling Technologies). Secondary antibodies were from Invitrogen, unless indicated otherwise: goat anti-mouse-IgG $\mathrm{Ig}_{1}$-Alexa-Fluor-488; goat anti-mouse$\mathrm{IgG}_{2 \mathrm{a}}$-Alexa-Fluor-488; goat anti-mouse-IgG $\mathrm{Ig}_{2 \mathrm{~b}}$-Alexa-Fluor-488; donkey antirabbit-Alexa-Fluor-488; donkey anti-goat-Alexa 488; goat anti-mouse- $\mathrm{IgG}_{1}-$ Alexa-Fluor-594; goat anti-mouse- $\operatorname{IgG}_{2 \mathrm{a}}-$ Alexa-Fluor-594; goat anti-mouse $\mathrm{IgG}_{2 \mathrm{~b}}$-Alexa-Fluor-594; donkey anti-rabbit-Alexa-Fluor-594; and goat antihamster-Alexa-Fluor-594. Immunostaining for cleaved caspase-3 and p63 was detected using an horseradish peroxidase (HRP)-conjugated secondary antibody (EnVision kit; DAKO, Carpinteria, CA) in combination with either DAB (EnVision kit) or Alexa-Fluor-488-tyramide (1:120, TSA kit, Invitrogen). Specificity of p63 immunostaining using this method was confirmed by staining lung tissue of $\operatorname{Trp} 63^{-/}$mice (Fig. 7) and data not shown. Staining for PHH3 was performed with either the EnVision kit or with Alexa-Fluor-conjugated donkey anti-rabbit antibodies (Invitrogen). The mounting medium was either permount (Fisher Scientific) or Fluoromount G (Southern Biotech, Birmingham, AL) with nuclear counterstain DAPI (1:2000, Sigma-Aldrich). Photomicrographs were captured using either Zeiss Observer.Z1 inverted fluorescent microscope (Carl Zeiss Inc) or Olympus Provis AX70 microscope (Olympus), and images were processed in AxioVision Release 4.6.3 software (Carl Zeiss Inc)

\section{Analysis of cellular proliferation}

Epithelial cell proliferation was determined by counting the number of PHH3immunoreactive nuclei as a function of total DAPI-positive nuclei within epithelial cells of either alveolar or conducting airways. Proliferative index (PI) was calculated as follows: PI $(\%)=($ number of PHH3-positive cells/number of counted cells) $\times 100$. The average PI was determined for at least 2000 cells counted from each of three representative lung samples from each genotype for alveolar and nonrecombined conducting airway, and at least 500 cells for recombined bronchiolar epithelium from $\mathrm{Cr}(+)$ mice. The difference in PI between each group was analyzed by $t$-test, with $P<0.05$ considered to be significant.

\section{Microarray analysis}

RNA was isolated from the left lobe including the primary bronchus of each of four adult male wild-type and SFTPC-Cre(+)-Ctnnbl ${ }^{(e x 3) f f l o x}$ lungs by acid guanidinium thiocyanate-phenol-chloroform extraction as described previously
(Chomczynski and Sacchi, 1987). RNA was quantified on a spectrophotometer (ND-1000, NanoDrop Technologies, Inc., Wilmington, DE), and a probe was synthesized from $2 \mu \mathrm{g}$ of total RNA by the Duke University microarray core facility. Probes were hybridized to Affymetrix mouse genome 4302.0 arrays (Affymetrix, Santa Clara, CA), and data annotated with Affymetrix Expression Console software. Differentially expressed genes were determined using the Scoregene software package (Jerusalem, Israel). Fold change was calculated by transforming raw data with a base 2 logarithm $\left(\log _{2} x\right)$ function and normalizing against the average. Significant gene expression changes were defined as fulfilling a Student's $t$-test value of $P<0.05$ and a threshold number of misclassifications (TNOM) of $\leq 1$. Gene expression heat maps were constructed using Treeview (ver. 1.60) software (Michael B. Eisen, University of California at Berkeley).

\section{Acknowledgements}

We thank Christina Burton and Lixia Luo for excellent animal husbandry and Makoto M. Taketo (Department of Pharmacology, Graduate School of Medicine, Kyoto University, Yoshida-Konoecho, Japan) for Ctnnbl ${ }^{(e x 3) f l o x}$ transgenic mice. We are indebted to Brigid Hogan for her critical reading of this manuscript and insightful suggestions.

\section{Funding}

This work was funded by the National Heart, Lung, and Blood Institute (NHLBI) [grant numbers 1U01HL099997-01, HL064888, HL090146]. Deposited in PubMed Central for release after 12 months.

Supplementary material available online at http://jcs.biologists.org/lookup/suppl/doi:10.1242/jcs.092734/-/DC1

\section{References}

Blache, P., van de Wetering, M., Duluc, I., Domon, C., Berta, P., Freund, J. N., Clevers, H. and Jay, P. (2004). SOX9 is an intestine crypt transcription factor, is regulated by the Wnt pathway, and represses the CDX2 and MUC2 genes. J. Cell Biol 166, 37-47.

Chomczynski, P. and Sacchi, N. (1987). Single-step method of RNA isolation by acid guanidinium thiocyanate-phenol-chloroform extraction. Anal. Biochem. 162, 156159.

Clevers, H. (2006). Wnt/beta-catenin signaling in development and disease. Cell 127, 469-480.

Daniely, Y., Liao, G., Dixon, D., Linnoila, R. I., Lori, A., Randell, S. H., Oren, M. and Jetten, A. M. (2004). Critical role of p63 in the development of a normal esophageal and tracheobronchial epithelium. Am. J. Physiol. Cell Physiol. 287, C171C181

Driskell, R. R., Goodheart, M., Neff, T., Liu, X., Luo, M., Moothart, C., Sigmund, C. D., Hosokawa, R., Chai, Y. and Engelhardt, J. F. (2007). Wnt3a regulates Lef-1 expression during airway submucosal gland morphogenesis. Dev. Biol. 305, 90-102.

Duan, D., Yue, Y., Zhou, W., Labed, B., Ritchie, T. C., Grosschedl, R. and Engelhardt, J. F. (1999). Submucosal gland development in the airway is controlled by lymphoid enhancer binding factor 1 (LEF1). Development 126, 4441-4453.

Gontan, C., de Munck, A., Vermeij, M., Grosveld, F., Tibboel, D. and Rottier, R. (2008). Sox2 is important for two crucial processes in lung development: branching morphogenesis and epithelial cell differentiation. Dev. Biol. 317, 296-309.

Goss, A. M., Tian, Y., Tsukiyama, T., Cohen, E. D., Zhou, D., Lu, M. M., Yamaguchi, T. P. and Morrisey, E. E. (2009). Wnt2/2b and beta-catenin signaling are necessary and sufficient to specify lung progenitors in the foregut. Dev. Cell $\mathbf{1 7}$ 290-298.

Harada, N., Tamai, Y., Ishikawa, T., Sauer, B., Takaku, K., Oshima, M. and Taketo, M. M. (1999). Intestinal polyposis in mice with a dominant stable mutation of the beta-catenin gene. EMBO J. 18, 5931-5942.

Li, C., Xiao, J., Hormi, K., Borok, Z. and Minoo, P. (2002). Wnt5a participates in distal lung morphogenesis. Dev. Biol. 248, 68-81.

Li, C., Li, A., Li, M., Xing, Y., Chen, H., Hu, L., Tiozzo, C., Anderson, S., Taketo, M. M. and Minoo, P. (2009). Stabilized beta-catenin in lung epithelial cells changes cell fate and leads to tracheal and bronchial polyposis. Dev. Biol. 334, 97-108.

Metzger, R. J., Klein, O. D., Martin, G. R. and Krasnow, M. A. (2008). The branching programme of mouse lung development. Nature 453, 745-750.

Mills, A. A., Zheng, B., Wang, X. J., Vogel, H., Roop, D. R. and Bradley, A. (1999) p63 is a p53 homologue required for limb and epidermal morphogenesis. Nature 398, 708-713.

Morotti, R. A., Cangiarella, J., Gutierrez, M. C., Jagirdar, J., Askin, F., Singh, G., Profitt, S. A., Wert, S. E., Whitsett, J. A. and Greco, M. A. (1999). Congenital cystic adenomatoid malformation of the lung (CCAM): evaluation of the cellular components. Hum. Pathol. 30, 618-625.

Morrisey, E. E. and Hogan, B. L. (2010). Preparing for the first breath: genetic and cellular mechanisms in lung development. Dev. Cell 18, 8-23. 
Moses, K. A., DeMayo, F., Braun, R. M., Reecy, J. L. and Schwartz, R. J. (2001). Embryonic expression of an Nkx2-5/Cre gene using ROSA26 reporter mice. Genesis 31, 176-180.

Mucenski, M. L., Wert, S. E., Nation, J. M., Loudy, D. E., Huelsken, J., Birchmeier, W., Morrisey, E. E. and Whitsett, J. A. (2003). beta-Catenin is required for specification of proximal/distal cell fate during lung morphogenesis. J. Biol. Chem. 278, 40231-40238.

Mucenski, M. L., Nation, J. M., Thitoff, A. R., Besnard, V., Xu, Y., Wert, S. E., Harada, N., Taketo, M. M., Stahlman, M. T. and Whitsett, J. A. (2005). Betacatenin regulates differentiation of respiratory epithelial cells in vivo. Am. J. Physiol. Lung Cell Mol. Physiol. 289, L971-L979.

Murtaugh, L. C., Law, A. C., Dor, Y. and Melton, D. A. (2005). Beta-catenin is essential for pancreatic acinar but not islet development. Development 132, 46634674

Nguyen, H., Merrill, B. J., Polak, L., Nikolova, M., Rendl, M., Shaver, T. M., Pasolli, H. A. and Fuchs, E. (2009). Tcf3 and Tcf4 are essential for long-term homeostasis of skin epithelia. Nat. Genet. 41, 1068-1075.

Okubo, T. and Hogan, B. L. (2004). Hyperactive Wnt signaling changes the developmental potential of embryonic lung endoderm. J. Biol. 3, 11.

Okubo, T., Knoepfler, P. S., Eisenman, R. N. and Hogan, B. L. (2005). Nmyc plays an essential role during lung development as a dosage-sensitive regulator of progenitor cell proliferation and differentiation. Development 132, 1363-1374.

Que, J., Okubo, T., Goldenring, J. R., Nam, K. T., Kurotani, R., Morrisey, E. E., Taranova, O., Pevny, L. H. and Hogan, B. L. (2007). Multiple dose-dependent roles for Sox2 in the patterning and differentiation of anterior foregut endoderm. Development 134, 2521-2531.

Que, J., Luo, X., Schwartz, R. J. and Hogan, B. L. (2009). Multiple roles for Sox2 in the developing and adult mouse trachea. Development 136, 1899-1907.

Rajagopal, J., Carroll, T. J., Guseh, J. S., Bores, S. A., Blank, L. J., Anderson, W. J., Yu, J., Zhou, Q., McMahon, A. P. and Melton, D. A. (2008). Wnt7b stimulates embryonic lung growth by coordinately increasing the replication of epithelium and mesenchyme. Development 135, 1625-1634.

Rawlins, E. L., Clark, C. P., Xue, Y. and Hogan, B. L. (2009). The Id2+ distal tip lung epithelium contains individual multipotent embryonic progenitor cells. Development 136, 3741-3745.
Reynolds, S. D., Zemke, A. C., Giangreco, A., Brockway, B. L., Teisanu, R. M., Drake, J. A., Mariani, T., Di, P. Y., Taketo, M. M. and Stripp, B. R. (2008). Conditional stabilization of beta-catenin expands the pool of lung stem cells. Stem Cells 26, 1337-1346.

Shannon, J. M. (1994). Induction of alveolar type II cell differentiation in fetal tracheal epithelium by grafted distal lung mesenchyme. Dev. Biol. 166, 600-614.

Shu, W., Jiang, Y. Q., Lu, M. M. and Morrisey, E. E. (2002). Wnt7b regulates mesenchymal proliferation and vascular development in the lung. Development 129, 4831-4842.

Shu, W., Guttentag, S., Wang, Z., Andl, T., Ballard, P., Lu, M. M., Piccolo, S., Birchmeier, W., Whitsett, J. A., Millar, S. E. et al. (2005). Wnt/beta-catenin signaling acts upstream of N-myc, BMP4, and FGF signaling to regulate proximaldistal patterning in the lung. Dev. Biol. 283, 226-239.

Stripp, B. R., Reynolds, S. D., Boe, I. M., Lund, J., Power, J. H., Coppens, J. T., Wong, V., Reynolds, P. R. and Plopper, C. G. (2002). Clara cell secretory protein deficiency alters clara cell secretory apparatus and the protein composition of airway lining fluid. Am. J. Respir. Cell Mol. Biol. 27, 170-178.

Taranova, O. V., Magness, S. T., Fagan, B. M., Wu, Y., Surzenko, N., Hutton, S. R. and Pevny, L. H. (2006). SOX2 is a dose-dependent regulator of retinal neural progenitor competence. Genes Dev. 20, 1187-1202.

Tompkins, D. H., Besnard, V., Lange, A. W., Wert, S. E., Keiser, A. R., Smith, A. N. Lang, R. and Whitsett, J. A. (2009). Sox2 is required for maintenance and differentiation of bronchiolar Clara, ciliated, and goblet cells. PLOS ONE 4, e8248.

Wang, Z., Shu, W., Lu, M. M. and Morrisey, E. E. (2005). Wnt7b activates canonical signaling in epithelial and vascular smooth muscle cells through interactions with Fzd1, Fzd10, and LRP5. Mol. Cell. Biol. 25, 5022-5030.

Zemke, A. C., Teisanu, R. M., Giangreco, A., Drake, J. A., Brockway, B. L., Reynolds, S. D. and Stripp, B. R. (2009). beta-Catenin is not necessary for maintenance or repair of the bronchiolar epithelium. Am. J. Respir. Cell Mol. Biol. 41, 535-543.

Zhang, Y., Goss, A. M., Cohen, E. D., Kadzik, R., Lepore, J. J., Muthukumaraswamy, K., Yang, J., DeMayo, F. J., Whitsett, J. A., Parmacek, M. S. et al. (2008). A Gata6Wnt pathway required for epithelial stem cell development and airway regeneration. Nat. Genet. 40, 862-870. 